\title{
A Simple Approach of Low Back Pain
}

\author{
Hani Almoallim 1,2,3*, Samar Alwafi1,2, Khaled Albazli1,2, Manal Alotaibi1,2, Tuqa Bazuhair ${ }^{1,2}$ \\ ${ }^{1}$ Department of Medicine, Medical College, Umm Alqura University, Makkah, KSA \\ ${ }^{2}$ Alzaidi Chair of Research in Rheumatic Diseases, Medical College, Umm Alqura University, Makkah, KSA \\ ${ }^{3}$ Department of Medicine, Dr. Soliman Fakeeh Hospital, Jeddah, KSA \\ Email: $\underline{\text { hmmoallim@uqu.edu.sa }}$
}

Received 1 July 2014; revised 2 August 2014; accepted 3 September 2014

Copyright (C) 2014 by authors and Scientific Research Publishing Inc.

This work is licensed under the Creative Commons Attribution International License (CC BY).

http://creativecommons.org/licenses/by/4.0/

(c) (i) Open Access

\section{Abstract}

Low back pain (LBP) is primarily managed in general practice and commonly underestimated or misdiagnosed by physicians. This article presents comprehensive review for diagnosis and evaluation of LBP according to current clinical studies guidelines. Our objectives are to define LBP, to establish how to take a detailed history and how to physically examine it in order to enable physicians to make an appropriate differential diagnosis for LBP, and to identify relevant investigations and referrals of patients with LBP. The article first offers a quick description of inflammatory back pain then discusses the importance of screening red flag patients with LBP and the importance of its early detection. Finally, we summarize how to outline a primary plan for managing and treating LBP. The article is prepared in the format of question and answer to make it targeted and accessible.

\section{Keywords}

Low Back Pain, Rheumatology, History, Examination, Management, Inflammatory Back Pain

\section{What Is Back Pain?}

Low back pain (LBP) is defined as pain, muscle tension or stiffness localized below the costal margin and above the inferior gluteal folds, with or without leg pain [1]. It can be classified as a non-specific LBP, a serious condition, or as a radicular syndrome [2]. Classification of LBP as acute or chronic can be a useful aid for prognosis to guide management. It is often classified as acute (less than 6 weeks), sub-acute (6 - 12 weeks), and chronic (more than 12 weeks).

There is a wide acceptance that the management of LBP should begin in primary care. Therefore, all physicians should know how to make a complete assessment for patients having LBP to provide them with the most

\footnotetext{
${ }^{*}$ Corresponding author.
} 
appropriate management and referrals. Clinical assessment by history taking and clinical examination is the cornerstone in diagnosis of LBP with a restricted use of clinical imaging. Anatomical or physiological abnormalities cannot explain most of LBP cases. However, imaging studies are important to exclude serious causes of LBP such as malignancies and infections and other anatomical degenerative changes.

\section{Significance of Back Pain}

Non-specific LBP is now clearly recognized as a major public health problem. The symptom of LBP is the second most complaint after common cold. In 70\% of cases, LBP has no obvious etiology or a well-known pathogenesis [1].

\section{How Common Is It?}

Our review of the global prevalence of LBP shows that it is a major problem throughout the world and that it is most prevalent among females and persons ages 40 - 80 years [2]. Literature shows that there has been an increase in the reported lifetime prevalence of LBP to become as high as $84 \%$, and the in prevalence of chronic LBP which reached $23 \%$, with $11 \%-12 \%$ of the population progressing to a disability [1] [3] [4].

\section{Physician's Awareness of Back Pain}

The impact of back pain on society and economy is substantial since it is the most frequent cause of disability for people under the age of 45 [5]. In 2009, the National Institute of Health and Care Excellence in United Kingdome published guidelines for management of non-specific LBP. In 2010, the Institute made a study to assess the impact of these guidelines on the management of back pain within primary care. It was concluded that these guidelines have not been well applied in management of LBP in primary care, which could be due to the lack of awareness of these guidelines or to adherence to other ones [6].

\section{What Is the Common Prognosis of LBP?}

The long-term prognosis of LBP is generally good. In 2008, in Australian primary health care centers a cohort study was done on 973 patients with recent onset LBP to estimate the one-year prognosis and to identify the prognostic factors. This study found that $83 \%$ had mild or no pain, $86 \%$ had minimal or no disability at one year follow-up; however, only 72\% had completely recovered [6]. In 2010, a survey was done on Australian general practice physicians (GP) about the application of LBP management guidelines showed that although the guidelines discourage the use of imaging, over one-quarter of patients were referred for imaging [6], and while the guidelines recommend that initial care should focus on advice and simple analgesics, only $20.5 \%$ received advice and $17.7 \%$ of patients received analgesics. The analgesics provided were typically non-steroidal anti-inflammatory drugs (37.4\%) and opioids (19.6\%). This indicates that the usual care provided by GPs for LBP does not match the care endorsed in international evidence-based guidelines and may not provide the best outcomes for patients. The unendorsed care may contribute to the high costs of managing LBP, and possibly to the fact that some aspects of the care provided carry a high risk of adverse effects [7].

\section{How to Approach LBP?}

The specific etiology of LBP is still unidentified in most of the patients presenting to healthcare but listening to the patient gives the physician the best opportunity to identify the causes of LBP [8]. A full history and physical examination is essential in developing a diagnostic plan to identify the cause(s) of symptoms and administering a therapeutic regimen to relieve the pain. A focused history and physical examination are essential in evaluating patients presenting with LBP to assess them for serious symptoms of neurologic compromise, inflammatory, or medical conditions. Most of the patients can be evaluated by history and physical examination alone if the duration of back pain is less than one month [8] but a through history and physical exam can guide clinicians for further indicated diagnostic studies in serious underlying conditions (see Figure 1). In the majority of cases, the pain is self-limited so no specific treatment is required.

\section{How to Take the History?}

The history typically starts with a full analysis of pain by assessing the type of onset, site of pain and radiation, 


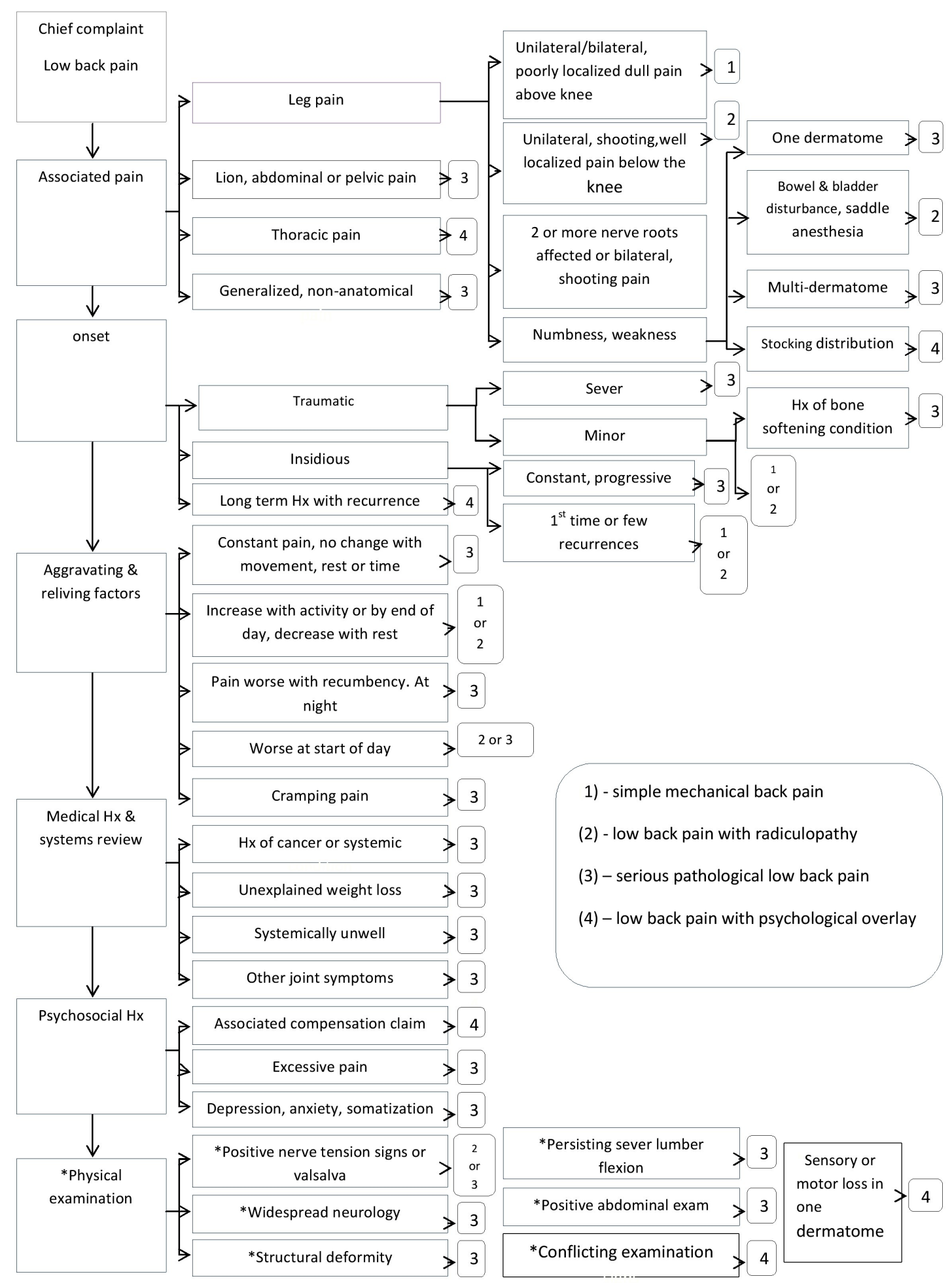

Figure 1. Approach to the patient with low back pain.

character and continuity of pain, progression and intensity of pain at rest and movement, factors altering pain, severity of pain, and associated symptoms. Type of pain may help the physician recognize the structure possibly injured, deep, nagging, and dull pain usually indicates the bones. A dull ache indicates muscles. A sharp and shooting pain indicates nerve root. A sharp, bright, lightning-like pain might be nerve. A burning, pressure-like, 
stinging, aching pain indicates sympathetic nerve. A throbbing diffused pain is mainly vascular. It is recommended in history taking to inquire about suggestive features of specific serious diagnosis associated with LBP like: cancer, infection, cauda equina syndrome, compression fractures, spinal stenosis, ankylosing spondylitis (AS), herniated disc or radiculopathy.

\section{What Are the Alarming Keys to Systemic Symptoms?}

Systemic symptoms in history taking may raise the suspicion of cancer or infection. Hence, it is important to pay attention to clues suggestive of underlying systemic diagnosis by asking about red flags such as any previous history of malignancy, unexplained weight loss, pain at night, and pain over four weeks with no response to treatment. These factors should be investigated properly for an age over 50 and the duration of pain over four to six weeks. The American College of Radiology has identified ten "red flags" in the LBP investigation that should be obtained to guide further recommended diagnostic imaging [9] (Table 1).

\section{What about Neurological Evidence?}

Symptoms of significant lumbar spinal stenosis include back pain, transient tingling in the legs, and ambulation-induced pain localized to the calf and distal lower extremity resolving with rest. Bowel or bladder dysfunction may be a symptom of severe compression, which calls for cauda equina syndrome. Urinary retention with overflowing incontinence is typically present with associated saddle anesthesia, bilateral sciatica, and leg weakness.

\section{How to Do Physical Examination?}

The paradigm "look, move, feel" should be utilized in the examination of LBP [10]. However, we have modified the terminologies used to describe the steps involved in the examination of LBP (see Figure 2). It starts typically by general inspection to observe the gait and posture looking for any asymmetry or abnormal curvature. Following this, a screening examination including the active range of motion (ROM) is performed. Detection of gross limitation of movement in ROM is less reliable in diagnosis. However, it can be recorded as a baseline to observe further progressions or improvement. A systematic approach to check palpation further is then undertaken by checking the palpation over each of the spinous processes, then unilaterally on each side of the process looking for underlying intervertebral segmental stiffness, tenderness and pain reproduction. This should be performed on each segment from the thoracolumbar junction to the lumbosacral junction. The examination is completed with checking palpation around the sacrum and buttock. The physical examination should finish with a detailed neurological evaluation and special tests like the straight leg raise test and/or slump test (see Figure 2 and Table 2). In young adults, it is recommended to perform straight leg raise test as it assesses possible sciatica. Elderly with spinal stenosis may have a normal straight leg raise test. A focused neurologic examination should be obtained particularly for ankle and knee reflexes, ankle and great toe to assess dorsiflexion strength, and distribution of sensory complaints to document the presence of neurologic deficits.

\section{When Should a Radiograph Be Used?}

Acute uncomplicated LBP without red flags is a benign self-limited condition that does not require imaging evaluation. In patients with red flags, MR has displaced CT and myelography as the initial imaging modality of choice in complicated LBP, with contrast useful for neoplasia, infection, and postoperative evaluation. However, CT is useful in patients with surgical fusion/instrumentation or bone structural abnormalities, and in patients with MRI contraindications. Myelography/CT, discography/CT, and radioisotope bone scans are useful in selected patients for problem solving.

\section{What Makes the Back Hurt?}

As back pain is a very common symptom it could be due to many causes, both specific and non-specific [1] as Table 3 shows.

\section{What Indicates an Inflammatory Back Pain?}

The diagnosis of inflammatory back pain (IBP) is often delayed in primary care. This may be due to inability to 
differentiate IBP from mechanical back pain. IBP can be a lifelong problem and it can impair function significantly. Its diagnosis is still a challenge within rheumatologic diseases where the diagnosis is delayed by 8 - 11 years from the onset of symptoms [11]. The longer the diagnosis is delayed, the worse the functional outcome

\section{Table 1. How to diagnose back pain by history and physical examination.}

History of presenting illness

- Onset: any significant or mild trauma preceding onset or insidious onset (AS).

- Duration: affects imaging and management decisions.

- Alleviating/exacerbating factors: positions, timing, past treatments, exercise and/or rest.

- Associated symptoms: sciatica, paresthesias, pseudoclaudication, hip/knee pain (inflammatory arthritis), bowel/bladder dysfunction.

Review of Systems:

- Visceral causes (Renal, GI, Pelvic).

- Systemic symptoms of cancer or infection: (Fever, weight loss, night sweat or loss of appetite).

Past medical and surgical history:

- Previous cancer history.

- Medications.

- Osteoporosis/pathologic fractures.

- Anxiety or depression.

Social History:

- Smoking, obesity, older age, intravenous (IV) drug use and work ergonomics.

- Inspect back and posture for any anatomical abnormalities.

- Palpate the back to assess vertebral or soft tissue tenderness (sensitive for spinal infection).

- Straight leg raise test to confirm radiculopathy.

- Neurologic assessment of L5 and S1 roots for patient suspected to have disc herniation.

- Evaluation for malignancy if history compatible with systemic diseases.

- Detect the baseline range of motion for the patient.

Red Flags appropriate for imaging:

- Recent significant trauma, or milder trauma age $>50$.

- Unexplained weight loss.

- Unexplained fever.

- Immunosuppression.

- History of cancer.

- IV drug use.

- Osteoporosis, prolonged use of glucocorticoids.

- $\quad$ Age $>70$.

- Focal neurologic deficit progressive or disabling symptoms.

- Duration greater than 6 weeks.

- Prior surgery.

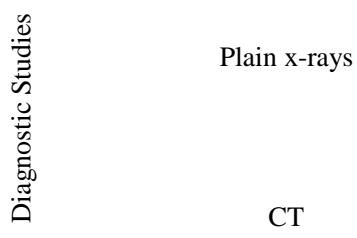

MRI

Electromyography (EMG)

Radionuclide bone scans
- Can detect infection, fracture, malignancy, spondylolisthesis, degenerative changes, disc space narrowing, and prior surgery.

- ESR can be used as a screening test if malignancy and infection are concerned, where are very unlikely in patients with an ESR $<20$ and no more than one risk factor for a systemic illness.

- Demonstrating bony abnormalities such as sacroiliac joint disease, fractures, spondylolisthesis, unstable fusions, abnormal facet joints, degenerative changes, and congenital abnormalities.

- Abnormal radiographs of the spine or non-diagnostic following trauma.

- Best for viewing soft tissues-indicated with neurologic signs/symptoms; most useful when there is concern for disk herniation, spinal stenosis, osteomyelitis, discitis, spinal epidural abscess, bone metastasis, arachnoiditis, neural tube defects.

- Detect the sacroiliac changes of AS before these are apparent on plain radiographs.

- For patients with radiculopathy who may be surgical candidates and who have poor correlation between their radicular symptoms and neuroimaging.

- For patients with multilevel disease evident on neuroimaging.

- Sensitive for detecting occult infection or a neoplasm than are plain radiographs.

- Limited use in patients who have both normal plain films and a normal ESR. 
Table 2. Lower back pain causes and risk factors.

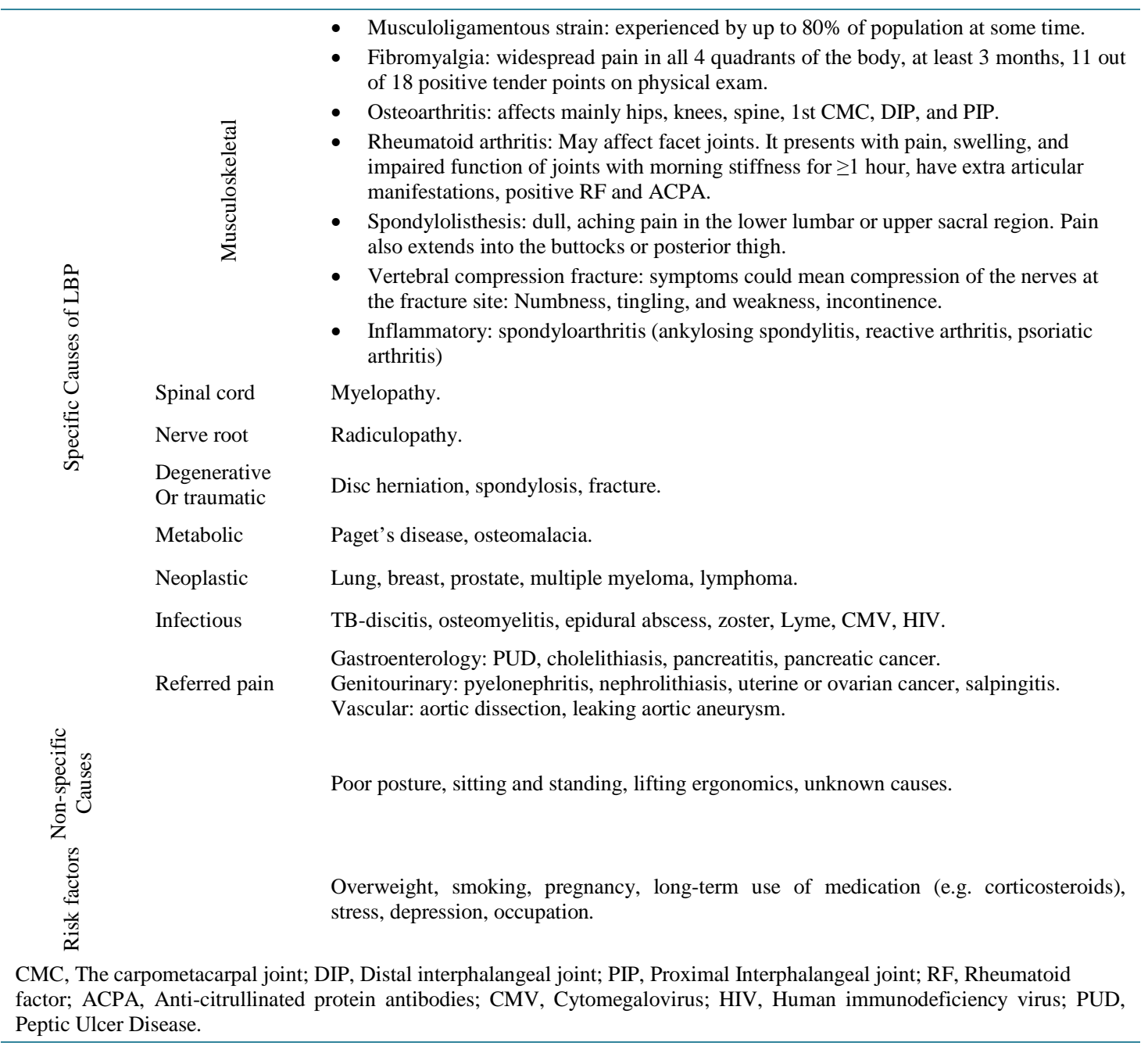

may be while an early diagnosis and treatments can result in achieving major improvements. AS is an important and often under recognized cause of LBP particularly for those who participate in sports [12].

Individual criteria used to define the presence of IBP which had a sensitivity of $77.0 \%$ and specificity of $91.7 \%$ are: improvement with exercise, pain at night, insidious onset, age at onset $<40$ years, and no improvement with rest [13]. If four out of these five parameters are fulfilled, the physician may consider it an IBP and refer the patient to a rheumatologist [13] [14]. IBP have variant clinical presentation (see Figure 3), the symptoms might present as skeletal manifestations e.g.: axial arthritis, enthesitis, osteoporosis, vertebral, fractures, spondylodiscitis, pseudoarthrosis or as extra skeletal manifestations e.g.: acute anterior uveitis, cardiovascular involvement, pulmonary involvement, cauda equina syndrome, enteric mucosal lesions [15].

\section{How to Detect LBP?}

Patients with complaints about bones and joints are often ignored and usually underestimated by doctors. Following the algorithm (Figure 4) can help to detect early back pain conditions [8].

\section{What Is the Treatment?}

There are many guidelines for therapeutic recommendations for non-specific back pain that are based on literature such as the ones done by the National Health Service in the United Kingdom, in 2008, the one by the American College of Physicians and the American Pain Society in the united states in 2007, or the one by the 

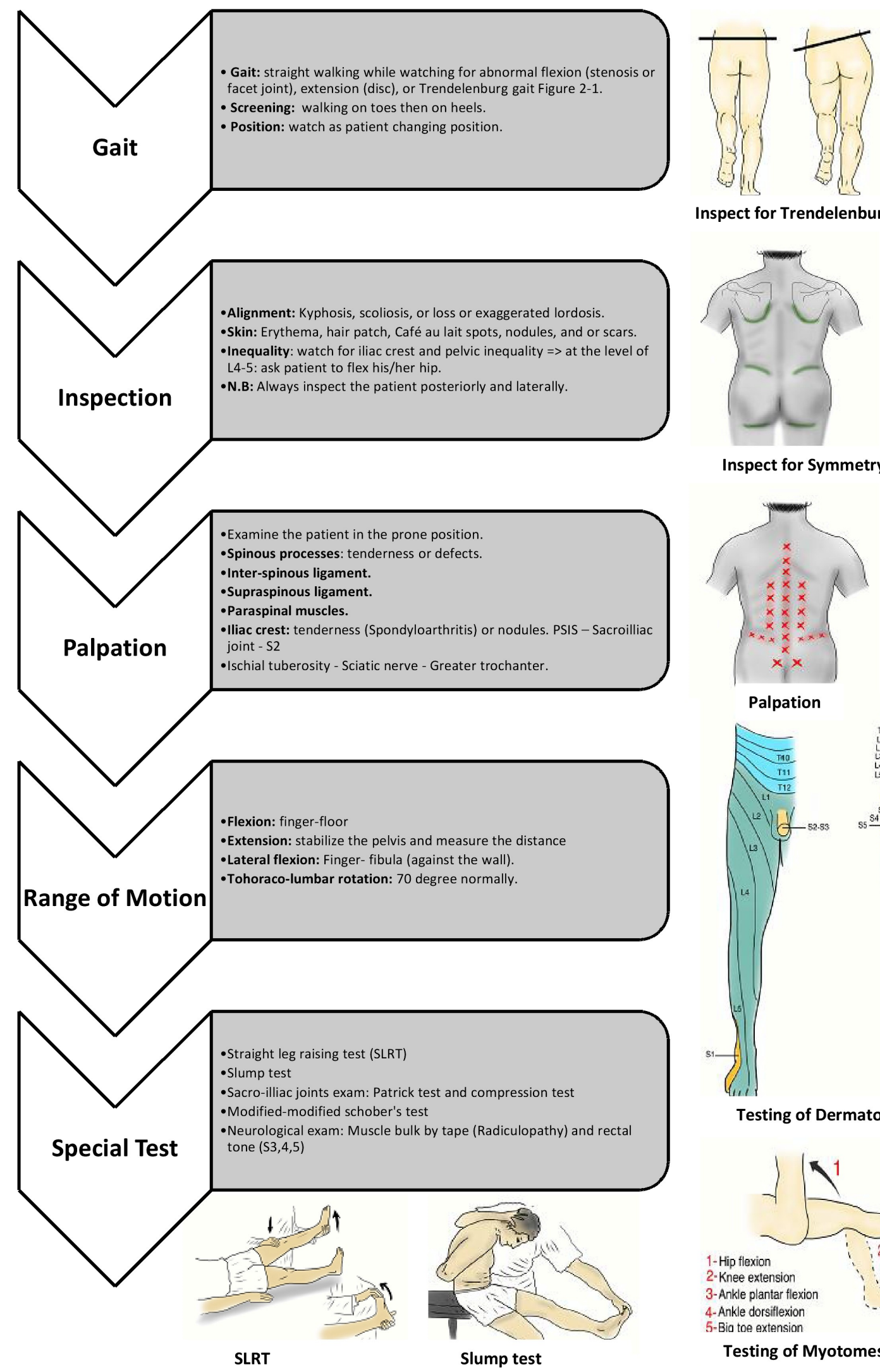

Inspect for Trendelenburg gait

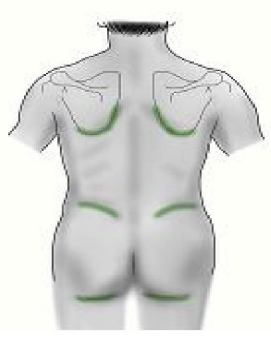

Inspect for Symmetry
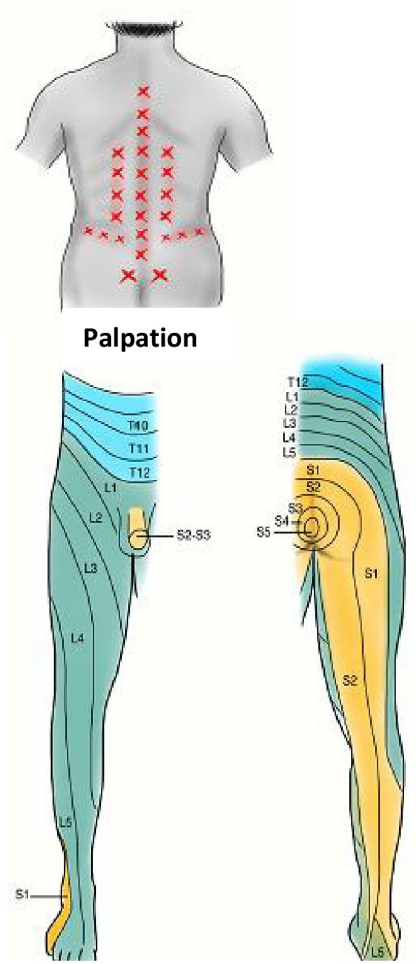

Testing of Dermatomes

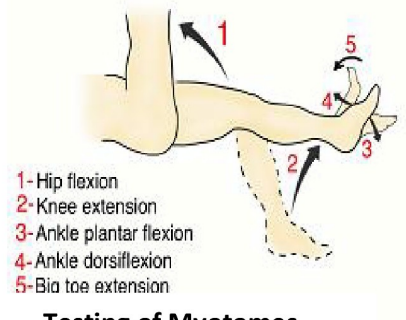

Testing of Myotomes

Figure 2. Physical examination for low back pain. 
Table 3. Special tests for LBP.

Special test

Straight leg raise test (SLRT)

Slump Test

Patrick’s Test (FABER) Test

Compression Test

Modified-modified Schobers Test

Neurological assessment

\section{Technique and significance}

- To test for the presence of a disc herniation.

- In supine position, flex the patient's hip while maintaining the knee in full extension.

- Watch for the degree of hip flexion where the patient reports pain.

- Positive SLRT: Radicular pain down the posterior portion of the tested leg at 40 degrees of hip flexion or less.

- Sensitivity 33\%, Specificity $87 \%$.

- Ask the patient to hold hands behind his/her back while seated upright.

- Instruct to the patient to (slump) flex his/her spine, followed by neck flexion.

- With examiner's hand on top of head, the patient performs knee extension, and dorsiflexion of foot.

- Ask the patient to return the neck to neutral (no flexion).

- Positive Slump test: The patient's symptoms are increased in the slumped position and released as the patient actively extends.

- Sensitivity $84 \%$, Specificity $83 \%$.

- To assess for the sacroiliac joint dysfunction or hip joint pathology.

- In supine position, bring the tested leg to hip flexion, abduction, and external rotation.

- Against the medial knee, try to bring the bent leg passively towards the table.

- Positive test: reproduction of groin pain or buttock pain.

- Sensitivity $82 \%$

- To assess for the sacroiliac joint dysfunction.

- While standing behind the patient, bring him/her to sideline position.

- Ask the patient to flex hip at 60 degrees and knees at 90 degrees.

- Apply an inward/downward force on iliac crest.

- Positive test: Pain on sacroiliac joint.

- Sensitivity $69 \%$ and Specificity $93 \%$.

- Identify the PSISs by marking the inferior margins of the patient's PSISs with his or her thumbs.

- Mark along the midline of the lumbar spines horizontal to the PSISs.

- Make another mark $15 \mathrm{~cm}$ above the original mark.

- With a tape pressed firmly on the line between the two marked points, instruct the patient to bend forward into full lumbar flexion.

- Measure the new distance between the superior and inferior skin markings.

- Distance increases less than $5 \mathrm{~cm}$ indicates limited lower back flexion.

- Measure the muscle bulk by tape.

- Assess for muscle power: Hip flexion (L2), knee extension (L3), ankle dorsiflexion (L4), big toe extension (L5), and ankle plantar flexion (S1).

- Check knee reflex (L3 and L4), and ankle reflex (L5 and S1).

- Check for skin sensory loss.

- Assess anal sphincter tone by digital examination $(\mathrm{S} 3,4,5)$. 


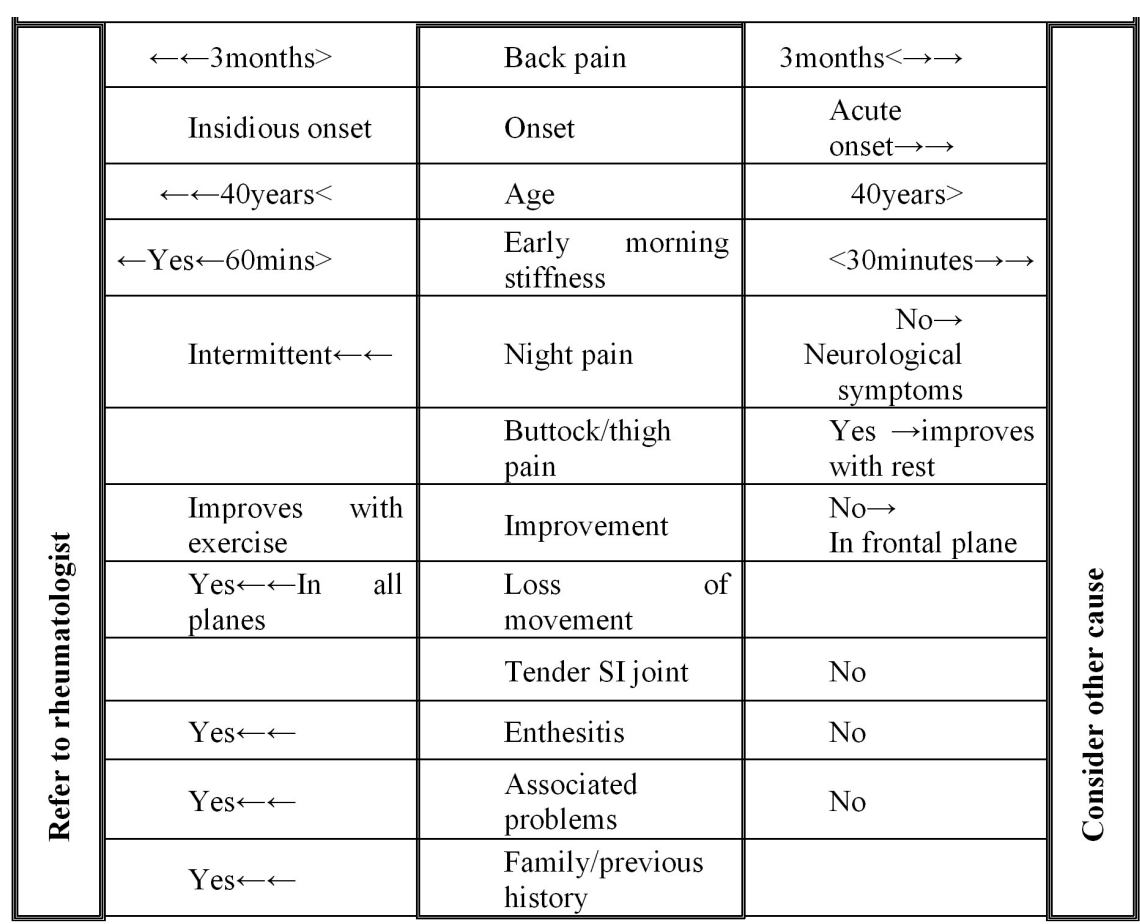

Figure 3. Diagnostic diagram of inflammatory back pain.

Clinic on Low back Pain in Interdisciplinary Practice in Canada 2007 [16] [17].

Patient advice and information was recommended in all guidelines. Reassurance of the patients and that they do not have a serious problem is the common message. Advising them to stay active and increasing their activity levels progressively. The current guidelines recommend early return to work (despite having LBP).

Paracetamol (acetaminophen) is generally recommended as a first choice of medication prescriptions due to its lower gastrointestinal side-effects incidence. In the case where paracetamol is not sufficient, non-steroidal anti-inflammatory drugs (NSAIDs) are the second choice. There is a variation between recommendations regarding opioids, muscle relaxants, steroids, antidepressant and anticonvulsive medication; to use it as co-medication to relief pain [18].

All guidelines discourage bed rest for LBP. In the case of severe pain some guidelines advise bed rest but for no longer than 2 days. There is consensus regarding exercising and physical activity, which is indicated in sub-acute and chronic pain but not considered in acute LBP. There is no evidence that a specific form of exercise is superior to another (Table 4).

\section{How Can Inflammatory Back Pain Be Treated?}

In IBP, the major aim for the treatment is the preservation and gain of short and long-term health in relation to the quality of life. The general view is that this is best achieved thorough control of symptoms and inflammation with the aim to prevent deformity and disability. Patient education and regular exercise is the cornerstone of non-pharmacological treatment. Supervised group exercises preferred because they are more effective than individual home exercises. IBP patients should be treated according to the disease manifestation of symptoms and signs: entheseal, axial, peripheral, or extra-articular, the general clinical status: age, gender, comorbidity, concomitant medications, psychosocial factors, and the level of current symptoms, clinical findings, and prognostic indicators. The first-line treatment is NSAIDs including cyclooxygenase-II inhibitors for patients who have symptoms and persistently active. Side effects of NSAID should be considered. If NSAID failed, contraindicated or not tolerated, paracetamol and opioid are recommended.

There is no evidence that disease-modifying anti-rheumatic drugs (DMARDs) including sulfasalazine, methotrexate and systemic glucocorticoids are effective for axial disease. In this case, corticosteroid injections to the site of musculoskeletal inflammation might be considered. 
Patient's complaints of LBP

Focused Hx\& PE: duration of symptoms, risk factors, symptoms of radiculopathy or

spinal stenosis, presence severity of neurologic deficit, psychological risk factors
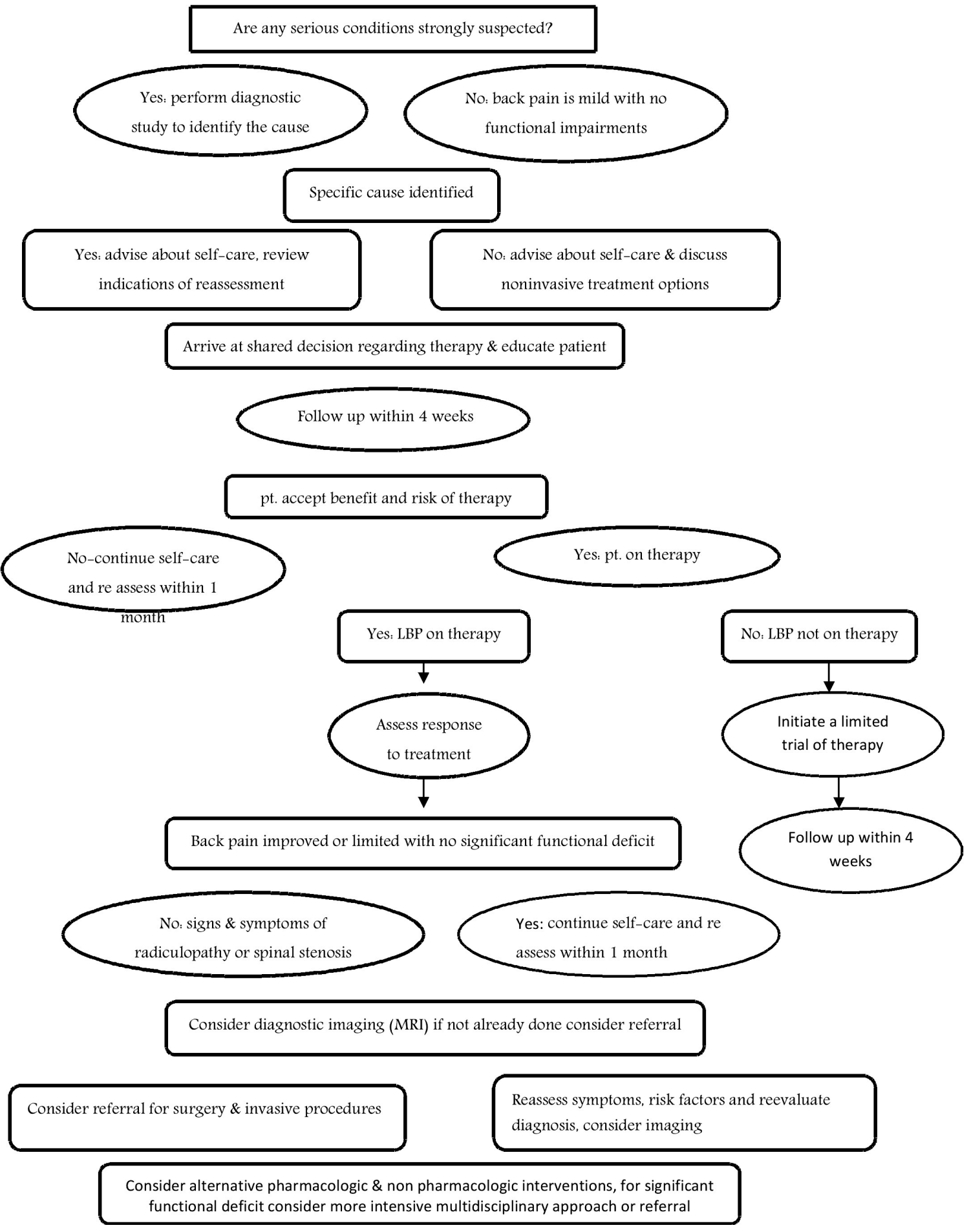

Figure 4. Diagnostic diagram of inflammatory back pain. 
Table 4. Summary of common recommendations for treatment of low back pain (acute or subacute pain).

- $\quad$ Reassure patients (favorable prognosis).

- $\quad$ Advise to stay active.

- $\quad$ Prescribe medication if necessary (preferably time-contingent).

- First line is paracetamol; second line is non-steroidal-anti-inflammatory drugs, consider muscle relaxants, opioids or antidepressant and anticonvulsive medication (as co-medication for pain relief).

- Discourage bed rest.

- Discourage use of modalities (such as ultrasound, electrotherapy).

- $\quad$ Short-term use of medication/manipulation.

- $\quad$ Supervised exercise therapy.

- Cognitive behavioral therapy.

- $\quad$ Multidisciplinary treatment.

In patients with a highly active disease, anti-TNF therapy is recommended. For patients with disability, refractory pain and radiological structural damage, surgical treatment should be considered, e.g. total hip arthroplasty despite the age of the patient. In severely disabling deformities spinal corrective osteotomy is considered. Surgical consultation is recommended in acute vertebral fracture and AS [19].

\section{When Should a Patient Be Referred?}

Usually a patient should be referred to a neurosurgeon or orthopedist when he has any of the following signs or symptoms is present [10]:

1) Cauda equina syndrome: the patient complains of typical features like bowel and bladder dysfunction (urinary retention), saddle anesthesia, and bilateral leg weakness and numbness.

2) Spinal cord compression: cancer patients or those who have risk of spinal metastases may present as acute neurologic deficits, and need emergent evaluation for surgical decompression or radiation therapy.

3) Progressive or severe neurologic deficit patients should be referred to a neurologist if any neuromotor deficit that persists after four to six weeks of conservative therapy is present.

4) Persistent sciatica, sensory deficit, or reflex loss after four to six weeks in a patient with positive straight leg raise test, consistent clinical findings, and favorable psychosocial circumstances such as realistic expectations and absence of depression, substance abuse or excessive somatization.

\section{Acknowledgements}

We are grateful for the support and funding from Alzaidi Chair of Research in Rheumatic Diseases, Umm Alqura University, Makkah, Saudi Arabia. The authors would also like to thank Dr. Khadijah Bawazeer for editing the manuscript and Dr. Yasser Alshomrani for drawing the illustrations.

\section{References}

[1] Balagué, F., Mannion, A.F., Pellisé, F. and Cedraschi, C. (2012) Non-Specific Low Back Pain. Lancet, 19, 379.

[2] Chou, R., Qaseem, A., et al. (2007) Diagnosis and Treatment of Low Back Pain: A Joint Clinical Practice Guideline from the American College of Physicians and the American Pain Society. Annals of Internal Medicine, 147, 478-491. http://dx.doi.org/10.7326/0003-4819-147-7-200710020-00006

[3] Concannon, M. and Bridgen, A. (2011) Lower Back Pain: A Need for Thorough Assessment. Practice Nursing, 22, 458-463. http://dx.doi.org/10.12968/pnur.2011.22.9.458

[4] Hoy, D., Bain, C., Williams, G., March, L., Brooks, P., Blyth, F., et al. (2012) A Systematic Review of the Global Prevalence of Low Back Pain. Arthritis Rheumatology, 64, 2028-2037. http://dx.doi.org/10.1002/art.34347

[5] Hill, J.C., Whitehurst, D.G., Lewis, M., Bryan, S., Dunn, K.M., Foster, N.E., et al. (2011, Oct) Comparison of Stratified Primary Care Management for Low Back Pain with Current Best Practice (STarT Back): A Randomised Controlled Trial. Lancet, 29, 1560-1571. 
[6] Williams, C.M., Maher, C.G., Hancock, M.J., McAuley, J.H., McLachlan, A.J., Britt, H., et al. (2010) Low Back Pain and Best Practice Care: A Survey of General Practice Physicians. Archives of Internal Medicine, 170, 271-277. http://dx.doi.org/10.1001/archinternmed.2009.507

[7] Henschke, N., Maher, C.G., Refshauge, K.M., Herbert, R.D., Cumming, R.G., Bleasel, J., et al. (2008) Prognosis in Patients with Recent Onset Low Back Pain in Australian Primary Care: Inception Cohort Study. British Medical Journal, 337, Article ID: a171. http://dx.doi.org/10.1136/bmj.a171

[8] Bhangle, S.D., Sapru, S. and Panush, R.S. (2009) Back Pain Made Simple: An Approach Based on Principles and Evidence. Cleveland Clinic Journal of Medicine, 76, 393-399. http://dx.doi.org/10.3949/ccjm.76a.08099

[9] Henschke, N., Maher, C.G., Ostelo, RW., De Vet, H.C., Macaskill, P. and Irwig, L. (2013) Red Flags to Screen for Malignancy in Patients with Low-Back Pain. Cochrane Database of Systematic Reviews, 2, Article ID: CD008686.

[10] Dagenais, S., Tricco, A.C. and Haldeman, S. (2010) Synthesis of Recommendations for the Assessment and Management of Low Back Pain from Recent Clinical Practice Guidelines. Spine, J10, 514-529. http://dx.doi.org/10.1016/j.spinee.2010.03.032

[11] Sieper, J., van der Heijde, D., Landewé, R., Brandt, J., Burgos-Vagas, R., Collantes-Estevez, E., et al. (2009) New Criteria for Inflammatory Back Pain in Patients with Chronic Back Pain: A Real Patient Exercise by Experts from the Assessment of SpondyloArthritis International Society (ASAS). Annals of the Rheumatic Diseases, 68, 784-788. http://dx.doi.org/10.1136/ard.2008.101501

[12] Braun, J. and Inman, R. (2011) Clinical Significance of Inflammatory Back Pain for Diagnosis and Screening of Patients with Axial Spondyloarthritis. Annals of the Rheumatic Diseases, 69, 1264-1285. http://dx.doi.org/10.1136/ard.2010.130559

[13] Harper, B.E. and Reveille, J.D. (2009) Spondyloarthritis: Clinical Suspicion, Diagnosis, and Sports. Current Sports Medicine Reports, 8, 29-34. http://dx.doi.org/10.1249/JSR.0b013e3181967ac6

[14] Dincer, U., Cakar, E., Kiralp, M.Z. and Dursun, H. (2008) Diagnosis Delay in Patients with Ankylosing Spondylitis: Possible Reasons and Proposals for New Diagnostic Criteria. Clinical Rheumatology, 27, 457-462. http://dx.doi.org/10.1007/s10067-007-0727-6

[15] Kubiak, E.N., Moskovich, R., Errico, T.J. and Di Cesare, P.E. (2005) Orthopaedic Management of Ankylosing Spondylitis. Journal of the American Academy of Orthopaedic Surgeons, 13, 267-278.

[16] Braun, J., van den Berg, R., Baraliakos, X., Boehm, H., Burgos-Vargas, R., Collantes-Estevez, E., et al. (2011) 2010 Update of the ASAS/EULAR Recommendations for the Management of Ankylosing Spondylitis. Annals of the Rheumatic Diseases, 70, 896-904. http://dx.doi.org/10.1136/ard.2011.151027

[17] Chou, R. and Huffman, L.H. (2008) Medications for Acute and Chronic Low Back Pain: A Review of the Evidence for an American Pain Society/American College of Physicians Clinical Practice Guideline. Annals of Internal Medicine, 148, 247-248.

[18] Perianayagam, G.R., Newey, M. and Sell, P. (2013) NICE Guidelines in the Management of Non-Specific Back Pain in Primary Care: Are They Being Used? Bone Joint Journal, 95, 184.

[19] Koes, B.W., van Tulder, M., Lin, C.W.C., Macedo, L.G., McAuley, J. and Maher, C. (2010) An Updated Overview of Clinical Guidelines for the Management of Non-Specific Low Back Pain in Primary Care. European Spine Journal, 19, 2075-2094. http://dx.doi.org/10.1007/s00586-010-1502-y 
Scientific Research Publishing (SCIRP) is one of the largest Open Access journal publishers. It is currently publishing more than 200 open access, online, peer-reviewed journals covering a wide range of academic disciplines. SCIRP serves the worldwide academic communities and contributes to the progress and application of science with its publication.

Other selected journals from SCIRP are listed as below. Submit your manuscript to us via either submit@scirp.org or Online Submission Portal.
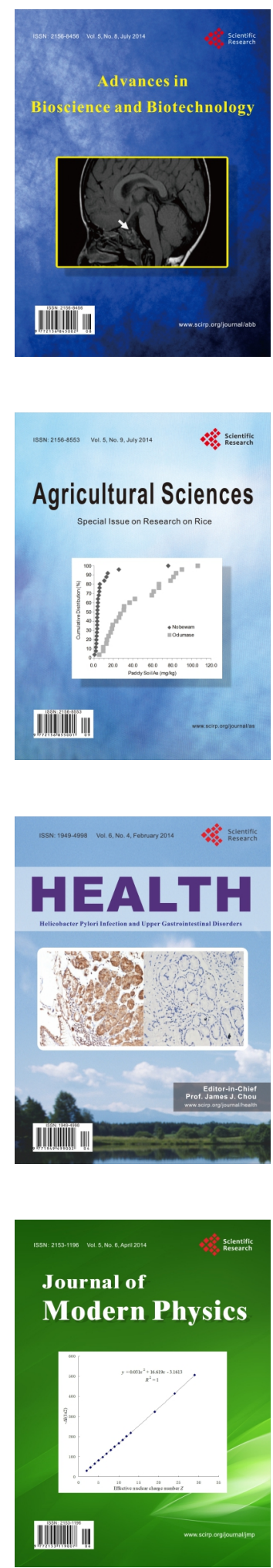
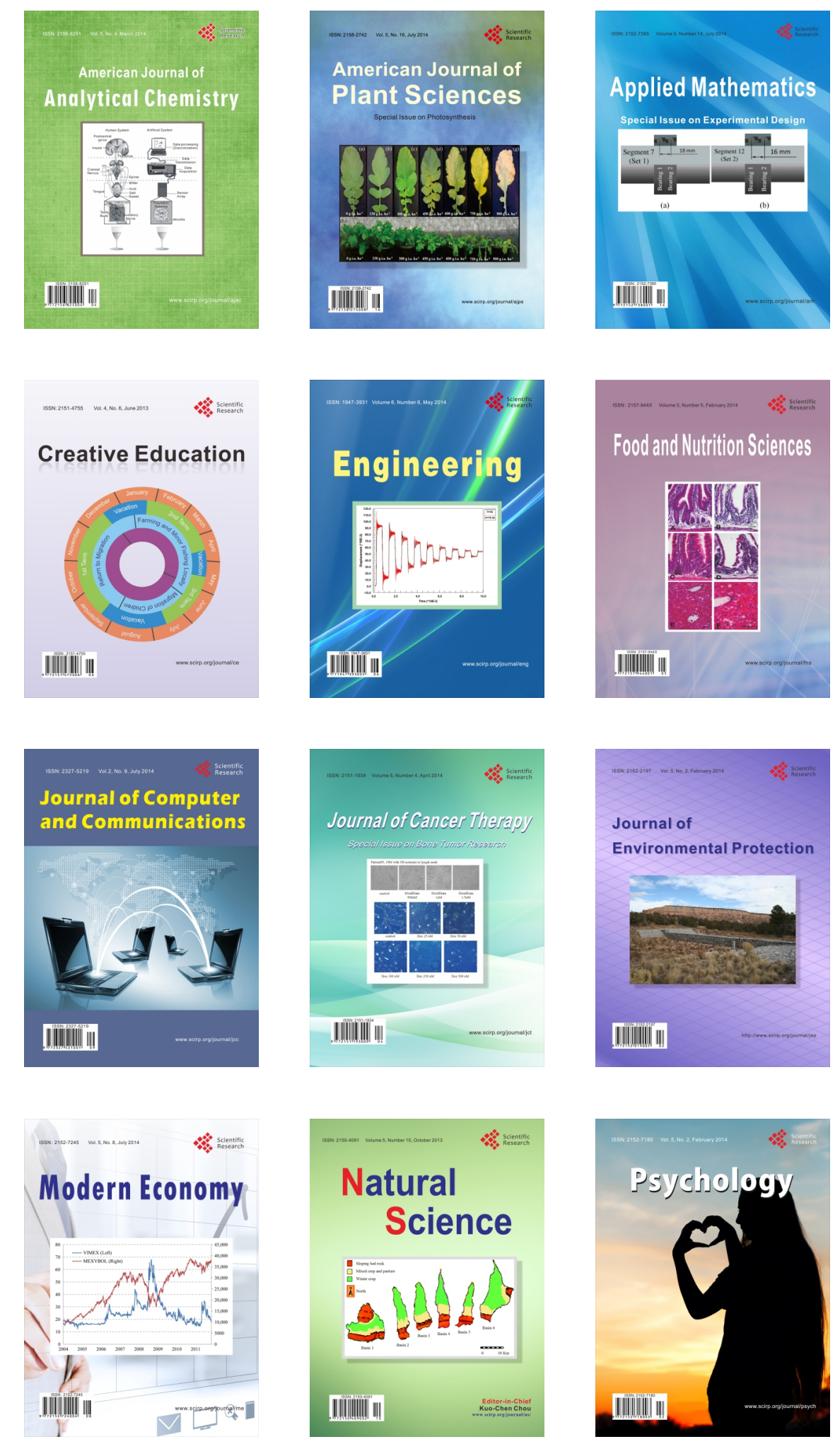